\title{
LA TEORÍA DEL CICLO VITAL DE FRANCO MODIGLIANI: ENFOQUE EN LOS SALARIOS DE LOS TRABAJADORES COLOMBIANOS
}

\author{
THE THEORY OF THE LIFE CYCLE OF FRANCO MODIGLIANI: \\ FOCUS ON THE SALARIES OF THE COLOMBIAN WORKERS
}

Juan Carlos Meza Martínez*

Recibido: 15 de agosto de 2017

Aprobado: 10 de noviembre de 2017

\section{Resumen}

La teoría de ciclo vital de Franco Modigliani estudia el salario, el ahorro y el consumo futuro en relación con la edad y el mercado laboral; esta teoría establece que hay un mayor ahorro en las primeras etapas de la vida y un desahorro al final del ciclo vital. Según el autor, la renta de las personas que se encuentran en el promedio de trabajadores que se retiran del mercado laboral tiende a disminuir en la medida que el trabajador envejece. En lo que respecta a la población ocupada, los hechos empíricos demuestran que el salario de los trabajadores aumenta hasta alcanzar un máximo, que cae después del retiro laboral, cumpliéndose de esta manera la teoría de Modigliani.

Palabras clave: ocupación, trabajadores, edad, salario.

\section{Abstract}

The life cycle theory of Franco Modigliani studies salary, savings and future consumption about age and the labor market. This theory establishes that there are more significant savings in the early stages of life and a dissaving at the end of the life cycle. According to the author, the income of the people who are in the average number of workers that retire from the labor market tends to decrease as the worker ages. Regarding the employed population, the empirical facts show that the wage of workers increases until reaching a maximum, which falls after retirement, thus fulfilling Modigliani's theory.

Keywords: occupation, workers, age, salary.

* Economista, magíster en Ciencias Económicas. Docente de cátedra, Fundación Universidad de América, Bogotá, Colombia.ORCID: https://orcid.org/0000-0001-9913-1198. juan.meza@profesores.uamerica.edu.co 


\section{INTRODUCCIÓN}

Una de las preocupaciones que tiene todo consumidor es maximizar sus salarios a lo largo de su vida laboral. En efecto, la hipótesis del ciclo vital desarrollada por Modigliani (1985) establece que los agentes deciden suavizar su consumo intertemporalmente para maximizar su salario; es decir, en las primeras etapas de su vida laboral los trabajadores ahorran para su vejez y luego desahorran en el consumo de sus necesidades.

Esta teoría puede aplicarse para el caso colombiano. Este artículo delimita el objeto de estudio a tres factores: vejez, salarios reales y niveles de educación; igualmente, estudia qué sucede con los trabajadores con salarios bajos en las primeras etapas de su vida y cómo este salario se incrementa en la medida que envejece el trabajador. Por consiguiente, se realizó un análisis descriptivo que explica el comportamiento del salario distribuido en las diferentes edades en promedio y se estimó un modelo econométrico de regresión por mínimos cuadrados ordinarios (MCO) y Quantile Regression, con los que se explican qué sucede con el salario por sexo y su respectivo nivel educativo.

Es importante tener en cuenta que la información con la que se pretende demostrar la hipótesis del ciclo vital se obtuvo de los microdatos de la Encuesta de Calidad de Vida (ECV) (Departamento Administrativo Nacional de Estadística, 2015) (esta encuesta no muestra el ahorro de las familias). Aquí se identificó a las personas con mayores salarios, con los cuales se puede mostrar la posibilidad de tener un mayor ahorro con respecto a aquellos que tienen un menor salario.

Este estudio analiza el enfoque del ciclo de vida de Franco Modigliani, enfatizando en la formación académica de los trabajadores colombianos.

Objetivos específicos:

- Determinar cómo los salarios reales de los colombianos caen con la edad (lo que valida la teoría del ciclo vital).

- Mostrar en cada cuantil de ingreso la pérdida salarial que se tiene en la medida que se avanza en edad.

- Determinar cuál es el salario real de los trabajadores en Colombia con cada nivel educativo alcanzado.

Este artículo se estructura en tres ejes temáticos: descripción de la teoría del ciclo vital; síntesis de los hechos empíricos en el análisis descriptivo de los salarios per cápita según género y niveles de educación (lo que demuestra que las personas con formación tardan más tiempo en el mercado laboral y que su salario solo cae casi a final de su vida), y resultados de la estimación del modelo.

\section{Teoría de ciclo vital de Modigliani, los salarios per cápita y los ahorros de los hogares}

Uno de los aspectos más importantes que define la hipótesis del ciclo vital está relacionado con las pérdidas y ganancias a lo largo de la vida laboral (Villar, 2012). Entre los trabajos que tratan sobre el ahorro y desahorro de los hogares se encuentra el de Melo, Zarate y Tellez (2006), el cual presenta el estado del arte sobre la suavización del consumo y realiza una interpretación teórica de la optimización intertemporal para describir una función de ganancias como resultado de un ciclo vital de ahorros que dura en los años productivos.

La decisión de ahorrar dependerá de lo alto que sean los salarios, ya sea en forma de pensión o de manera voluntaria. Por esta razón, el consumo futuro será tan grande como sean los salarios en las primeras etapas de la vida laboral. En este sentido, los trabajadores tendrán ahorros positivos toda vez que se encuentren en las primeras etapas de la vida, por su parte, en el periodo de jubilación los ahorros tienden a ser negativos (Melo, Zarate y Tellez, 2006). 
Cabe anotar que el crecimiento de los salarios no es lineal, al contrario, presenta efectos marginales (Hussein y Thirlwall, 1999): los trabajadores tendrán en algún momento de su vida laboral un máximo salario alcanzado que posteriormente desciende en forma de desahorro.

Asimismo, los salarios son producto de las capacidades formativas de los trabajadores, es decir, entre mayor calificación tengan los trabajadores, estos podrán acceder a mejoras salariales. Por lo cual, la propensión marginal al ahorro debe ser mayor en la medida que se tenga mayor nivel de renta (Mas-Colell, 1995) asociado a aumentos en la educación, los cuales implican un mayor bienestar después de la edad productiva.

Otro factor importante es el género, ya que debido a la estructura del mercado laboral existen diferencias entre los salarios de los hombres y mujeres. Esto también se verá reflejado en el ahorro.

En síntesis, los ahorros están sujetos, cuanto menos, a tres variables fundamentales que explican el consumo futuro: nivel de formación (que mejora la productividad y los salarios per cápita), efectos marginales de los salarios y diferencias entre hombres y mujeres. A continuación se demostrará que la formación es uno de los factores de producción que permite las mejoras salariales y, por consiguiente, el consumo presente y futuro.

\section{Ciclo vital, salarios y capital humano en Colombia}

De acuerdo con la información de la ECV 2015 del DANE, existe cerca de 22 millones de personas ocupadas en todos los rangos de edad. En el trabajo de Granados (2004) se afirma que entre los hechos empíricos la función de salarios per cápita ( $w)$ está determinado por los cambios en la edad, así como la pendiente de la curva de los salarios $\partial w / \partial e d a d=0$ y la segunda derivada $\partial^{2} w / \partial e d a d^{2}<0$. Sobre la base que los rendimientos de los salarios son decrecientes, un aumento en la edad reduciría $w$.

De acuerdo con Granados (2004), los salarios se estabilizan hacia el final del ciclo vital (efecto edad). Además, este estudio muestra la función de salarios como componente de medias móviles de primer y segundo orden, lo que demuestra el supuesto de la caída de los salarios con la edad. Asimismo, durante todo el ciclo, la magnitud de los salarios aumenta con la educación. La ECV 2015 comprueba que el avance en formación los trabajadores perciben unos salarios per cápita más altos (ver figura 1 ).

La figura 1 muestra que los cambios salariales, cuando hay un mayor nivel educativo, equivalen a un mejor bienestar y un mayor consumo futuro. Por su parte, McConell (2007) señala que el descenso de los salarios per cápita en las personas en la edad en que no participan en el mercado laboral se debe a dos factores: a) la educación formal y la capacitación en el trabajo las cuales van depreciándose con el tiempo y b) la disminución de los salarios per cápita de las personas de más de 65 años.

La propensión marginal a consumir a partir de la renta permanente y transitoria de Friedman (1976) es constante en todos los periodos de la vida, ya que el individuo tratará de mantener un consumo constante (Dornbusch, Fisher y Startz, 2009). Sin embargo, en la medida que el nivel de formación aumenta en los trabajadores, el consumo será mayor con respeto a aquellos que no invierten en capital humano.

En síntesis, la educación es una de las claves para el crecimiento salarial de los trabajadores colombianos y explica la propensión marginal al ahorro. La hipótesis del ciclo vital se cumple en el caso colombiano, por lo tanto, las personas con niveles altos de educación son más productivas y su permanencia en el mercado de trabajo es mayor con respecto a aquellas sin formación. A continuación, según datos estadísticos, se analizan los salarios por grupos de edad y niveles de escolaridad de los colombianos. 


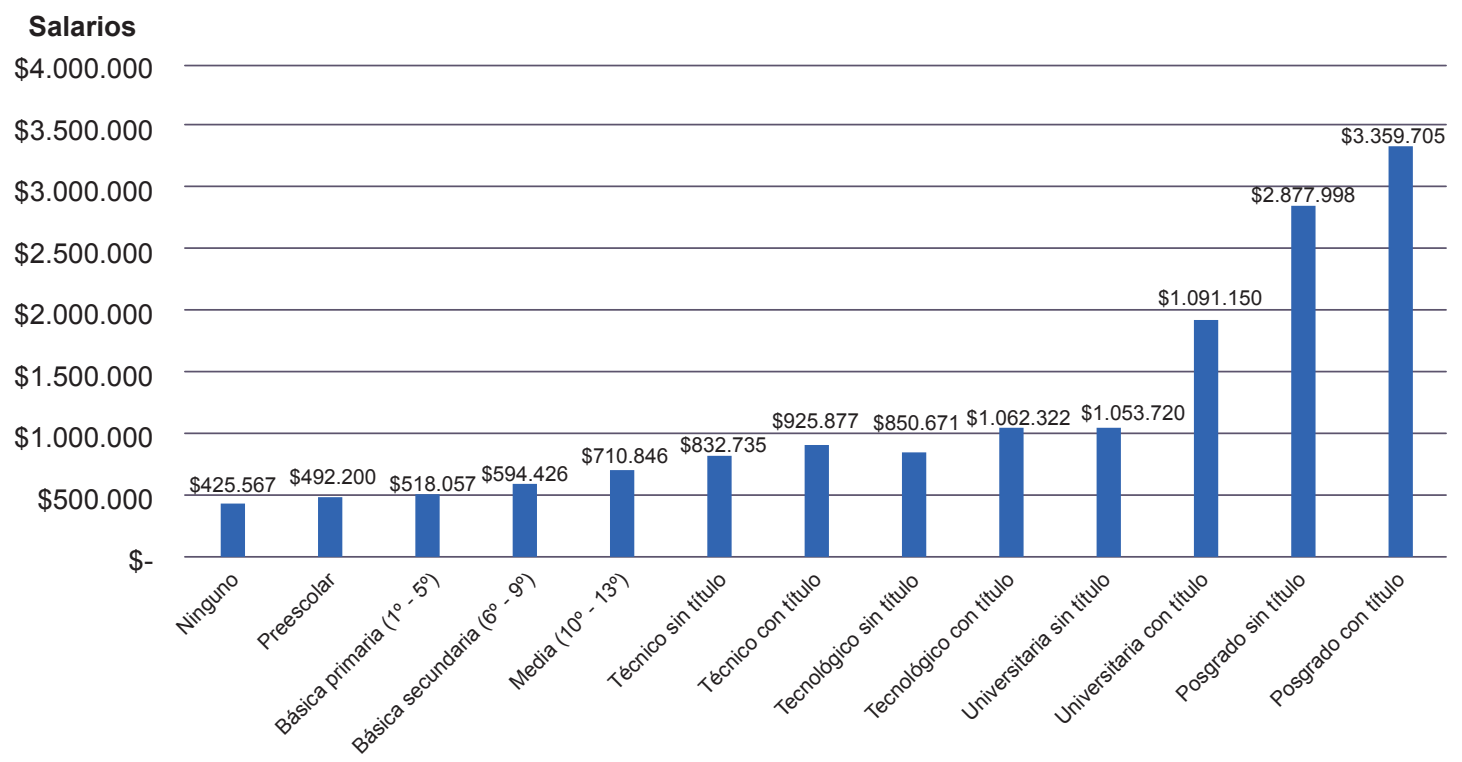

Nivel educativo

Figura 1. Gráfico de salario y años de educación.

Nota. La figura denota la relación entre los niveles educativos alcanzados en el eje de las abscisas y los salarios per cápita de los trabajadores colombianos en el eje de las ordenadas. Desde cero años de educación hasta posgrado con título.

Fuente. diseño de autor según Dane (2015).

\section{Salarios, educación y ciclo vital en Colombia}

En la figura 2 se aprecia que las personas a partir de los 65 años tienen salarios menores con respecto a los trabajadores con menos edad. Esto confirma lo señalado por Franco Modigliani, cuando asegura que los hogares desahorran después de la edad de la jubilación.

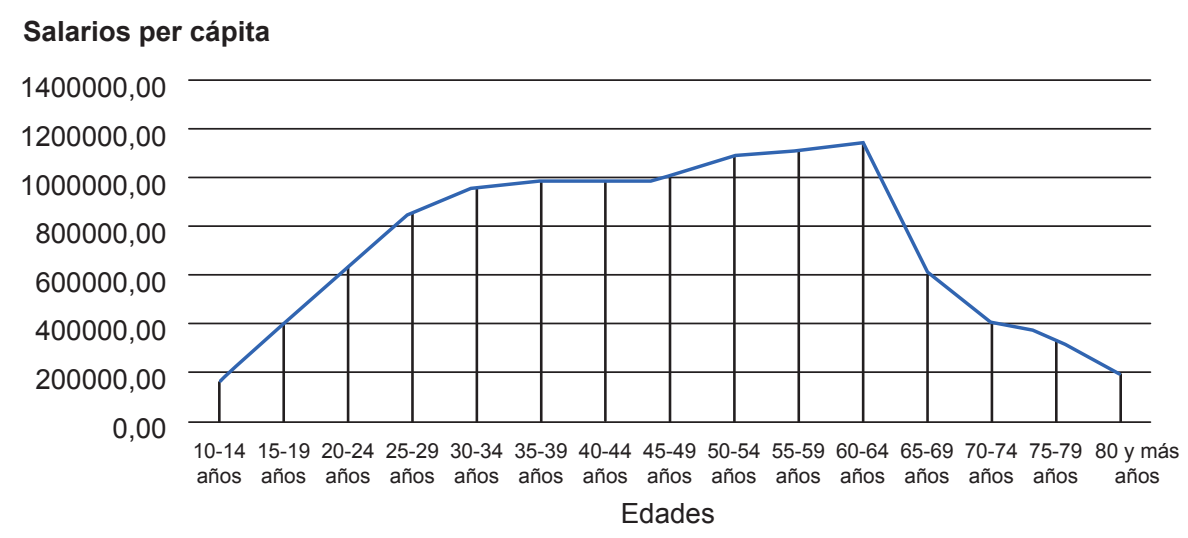

Figura 2. Gráfico de salarios per cápita y grupos de edades por quinquenios.

Nota. La figura muestra la relación entre los salarios per cápita de los trabajadores colombianos en el eje de las ordenadas y los grupos de edad. Nótese la figura presenta estadísticamente la forma de la distribución normal, lo que permite inferir que los datos están parametrizados, en los cuales puede existir una relación de dependencia lineal.

Fuente: diseñada por el autor según DANE (2015). 
En las primeras etapas de la vida los individuos tratarán de maximizar sus salarios. La forma cómo esto se logra es a través de la educación. Tal como se observa en la figura 3, los grados de educación permiten alcanzar salarios más altos de manera permanente en relación a niveles educativos más bajos.

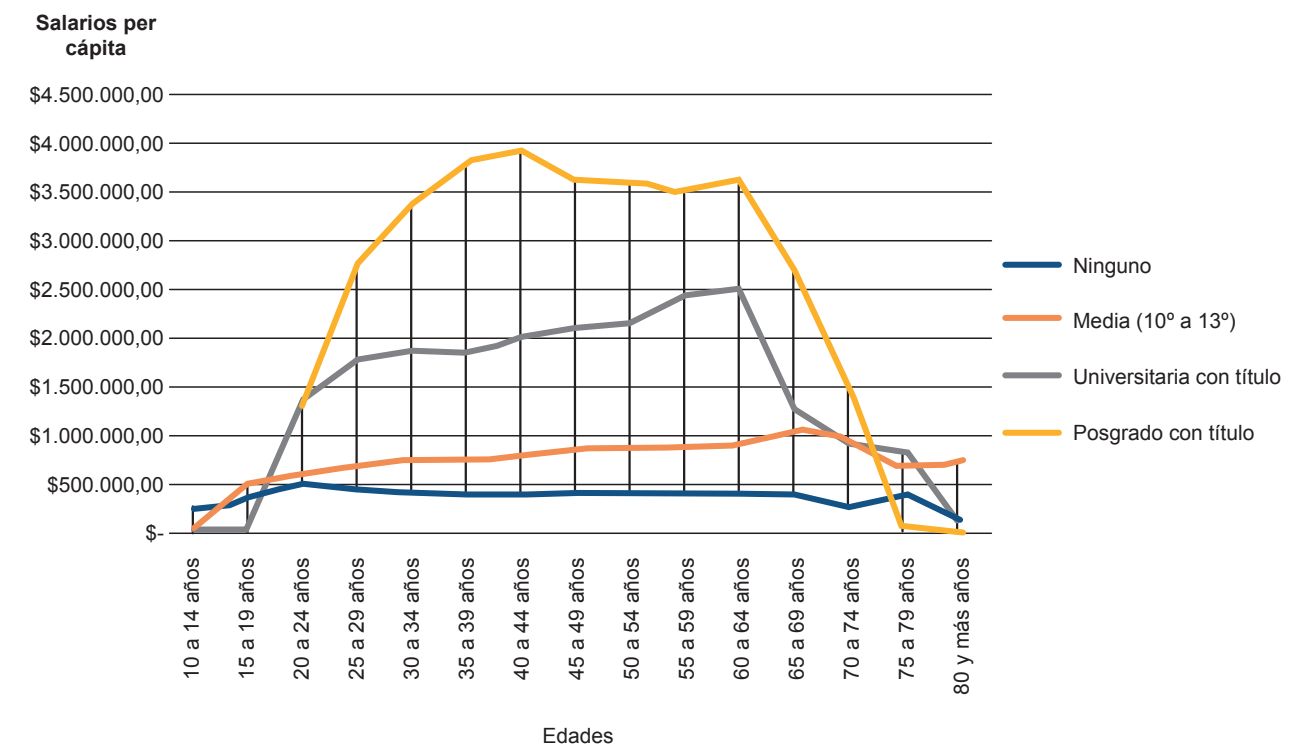

Figura 3. Gráfico de salarios, edad y educación en Colombia.

Fuente: diseño del autor según DANE (2015).

Además, se puede apreciar que los salarios de los trabajadores que no tienen ningún nivel educativo y aun en la educación media $\left(10^{\circ}-13^{\circ}\right)$ son menores con respecto a lo que tienen educación universitaria y títulos de posgrado. Estos se encuentran 400 veces más bajos que aquellos que tienen mayor formación. Entonces, la educación garantiza una mejora del bienestar en cuanto a renta disponible.

De acuerdo con el esquema de consumo y ahorro del ciclo vital de Modigliani (Dornbusch, Fisher y Startz, 2009), el comportamiento estadístico es muy similar a la hipótesis, dado que presenta las primeras etapas de la vida laboral de las personas conforme a su renta percibida por su trabajo (ver figura 4).

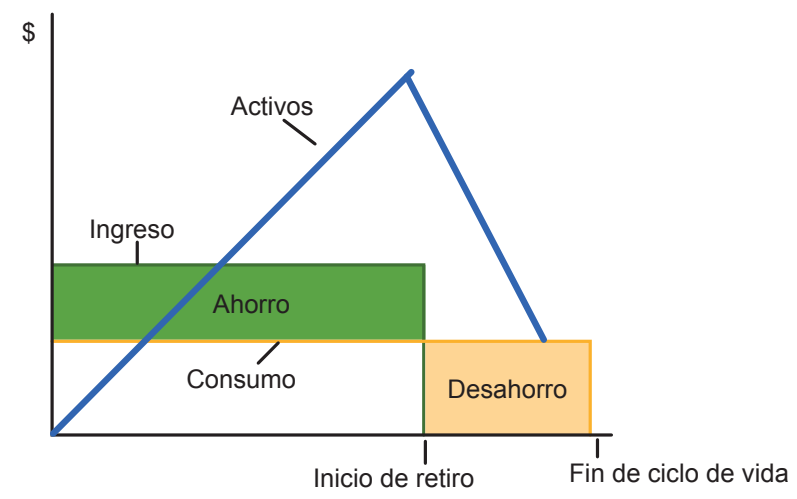

Figura 4. Modelo del ciclo vital.

Fuente: Dornbusch y Fisher (1998) 
Sin embargo, la hipótesis no toma en cuenta factores que discriminen los salarios a lo largo de la vida laboral como son los años de educación, el género y territorio; aspectos importantes en el momento de medir las variables. Asimismo, los resultados del modelo econométrico sobre la relación con los salarios las edades y sus grados de educación en Colombia se describen a continuación.

\section{Resultados del modelo econométrico de MCO y regresión cuantílica}

Los datos son tomados de los microdatos de la ECV 2015 del DANE. Por esta razón se utilizará el modelo MCO y Quantile regression; modelos que permiten estimar una función lineal en la que se toma la variable edad y se distribuye por rangos. La función a estimar por tramos de edad sería la siguiente:

$$
\ln w=\beta_{0}+\beta_{1} f\left(e d a d_{i}\right)+\beta_{2} f\left(e d u_{i}\right)+\beta
$$

Donde $\ln w$ es el logaritmo natural del salario. Además, se considera que la $e d a d_{i}$ se divide en los siguientes rangos por décadas:

- Edad 1: 18 a 28 años

- Edad 2:29 a 39 años

- Edad 3: 40 a 50 años

- Edad 4: 51 a 61 años

- Edad 5:62 a 72 años

- Edad 6: 72 años y más

La variable $e d u_{i}$ alude a los años de educación: ningún año de educación, bachillerato, profesional y posgrado. En estos niveles se observará que los salarios son menores en la medida en que la edad aumenta. Sin embargo, cuando se estudia, un año de educación aumenta significativamente los salarios.

En la tabla 1 se evidencia los salarios de hombres y mujeres en los primeros años de la vida laboral se encentran alrededor del $30 \%$ y $12 \%$, respectivamente. Sin embargo, estos alcanzan su máximo salario entre las edades de 40 a 50 (edad 3), años donde se encuentra el punto más alto.

Tabla 1. Regresión de MCO

\begin{tabular}{lcccccc}
\hline \multirow{2}{*}{ Variables } & \multicolumn{2}{c}{ Hombre } & \multicolumn{2}{c}{ Mujer } & \multicolumn{2}{c}{ Total } \\
\cline { 2 - 7 } & Coeficientes & $\mathbf{P}>|\mathbf{t}|$ & Coeficientes & $\mathbf{P}>|\mathbf{t}|$ & Coeficientes & $\mathbf{P}>|\mathbf{t}|$ \\
\hline edad1 & 0,111859 & 0.006 & 0.01909 & 0.687 & 0.072332 & 0.020 \\
edad2 & 0.341779 & 0.000 & 0.12616 & 0.007 & 0.242934 & 0.000 \\
edad3 & 0.311453 & 0.000 & 0.12745 & 0.012 & 0.228784 & 0.000 \\
edad4 & 0.272668 & 0.000 & 0.13888 & 0.019 & 0.221057 & 0.000 \\
edad5 & -0.147672 & 0.075 & -0.15859 & 0.233 & -0.144970 & 0.038 \\
edad6 & -0.510698 & 0.004 & -0.70626 & 0.064 & -0.533071 & 0.001 \\
Ningún años de educación & -0.351921 & 0.000 & -0.89639 & 0.000 & -0.443690 & 0.000 \\
bachillerato & 0.153604 & 0.000 & 0.01952 & 0.531 & 0.100263 & 0.000 \\
profesional & 0.816894 & 0.000 & 0.95680 & 0.000 & 0.864470 & 0.000 \\
posgrado & 1.432236 & 0.000 & 1.49940 & 0.000 & 1.436732 & 0.000 \\
cons & 13.033410 & 0.000 & 12.99130 & 0.000 & 13.020000 & 0.000 \\
\hline
\end{tabular}

Fuente: diseño del autor según DANE (2015). 
Entre las edades de 72 años y más (edad 6) se refleja que los hombres pierden el $51 \%$ de su salario y las mujeres el $71 \%$. Asimismo, en la figura 5 se puede apreciar como caen estos salarios con la edad.

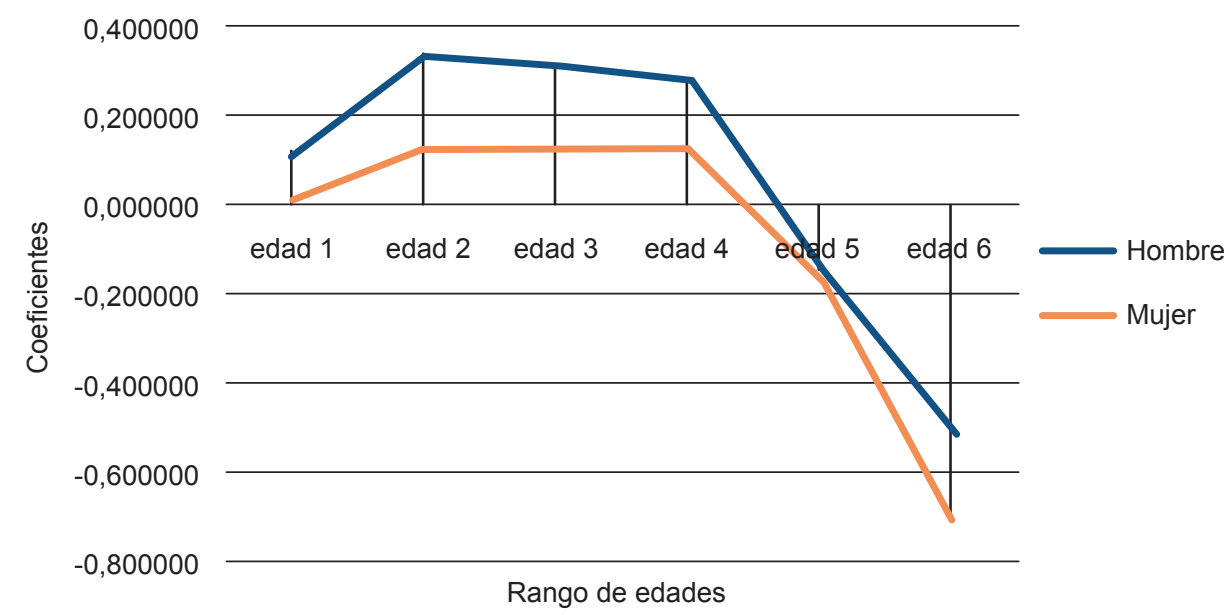

Figura 5. Gráfico de coeficientes de edades-MCO.

Nota. La figura muestra la pendiente de la curva de cada coeficiente, está se hace negativa en los rangos de edad más avanzados. Fuente: diseño del autor DANE (2015).

Por consiguiente, en edades avanzadas el salario se hace cada vez menor, lo que demuestra que los hombres gastan el $51 \%$ del salario y las mujeres el $71 \%$, esto se debe a que la propensión marginal al ahorro es menor en el género femenino debido a que su salario es menor con respecto al género masculino.

Con respecto a la educación, se puede observar en la figura 6 que el crecimiento en los salarios por cada nivel de educación aumenta considerablemente, principalmente aquellos que son mano de obra calificada y especializada.

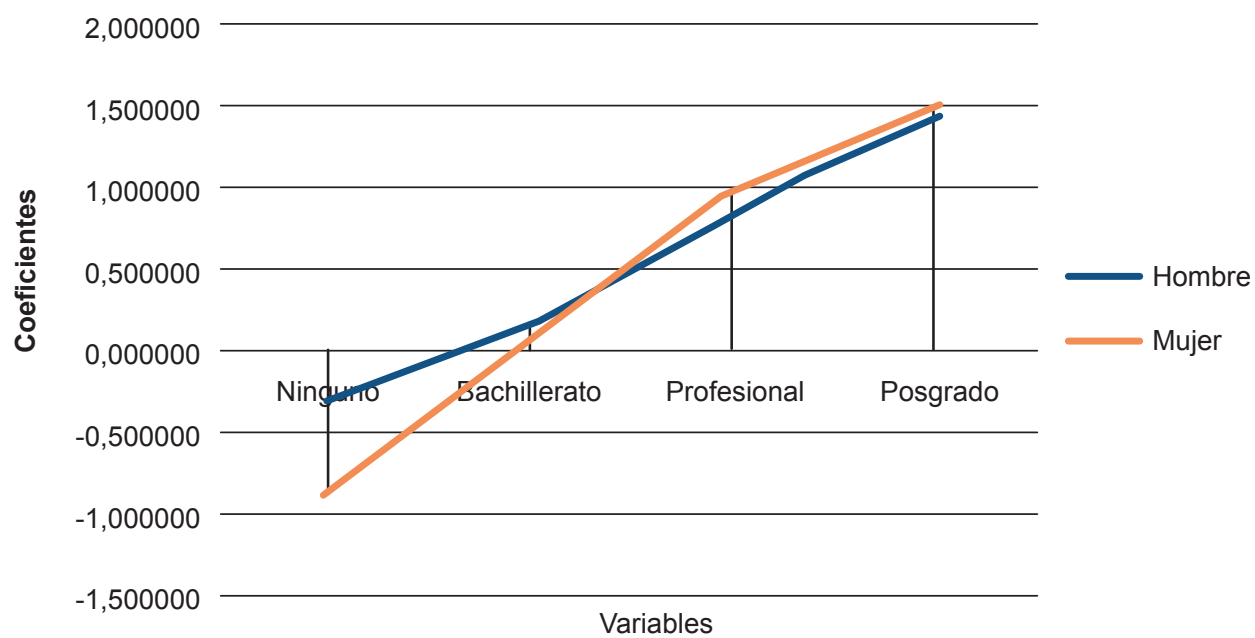

Figura 6. Gráfico de coeficientes de educación-MCO.

Fuente: diseño del autor según DANE (2015). 
Los salarios para profesionales y posgrado pueden llegar a tener cambios del $143 \%$; por esta razón la propensión marginal a ahorrar es mucho mayor con respecto aquellos que no tienen ningún nivel de educación o bachillerato. La siguiente regresión centra su análisis en cuantiles de ingresos, que es conocida como regresión cuantílica o quantile regression, con la que se analizó los perfiles de salarios entre hombres y mujeres.

\section{Regresiones cuantílicas para el caso colombiano}

En cuanto a la quantile regression se obtuvo tres perfiles de salarios con respecto al cuantil 25, 50 y 75. Aquí se observó que el $25 \%$ de las personas con menor salario presentan una pérdida de $59 \%$ de su salario. Asimismo, la pérdida de bienestar de las mujeres con respecto a su salario es del $48 \%$, mientras que el de los hombres es de $88 \%$ para las personas de más de 72 años (edad 6), así como lo muestra la tabla 2 .

Tabla 2. Modelo de regresión cuantílica

\begin{tabular}{|c|c|c|c|c|c|c|}
\hline \multirow{2}{*}{$\begin{array}{c}\text { Variables } \\
\text { Quantile } 25\end{array}$} & \multicolumn{2}{|c|}{ Hombre } & \multicolumn{2}{|c|}{ Mujer } & \multicolumn{2}{|c|}{ Total } \\
\hline & Coeficientes & $P>|t|$ & Coeficientes & $P>|t|$ & Coeficientes & $P>|t|$ \\
\hline edad1 & 0.2231436 & 0.003 & 0.1451826 & 0.042 & 0.2231436 & 0.000 \\
\hline edad2 & 0.4418325 & 0.000 & 0.2169123 & 0.003 & 0.450201 & 0.000 \\
\hline edad3 & 0.4418325 & 0.000 & 0.1451826 & 0.048 & 0.4076414 & 0.000 \\
\hline edad4 & 0.4054651 & 0.000 & 0.1715002 & 0.021 & 0.385663 & 0.000 \\
\hline edad5 & -0.3646431 & 0.030 & -0.2114925 & 0.388 & -0.1251631 & 0.229 \\
\hline edad6 & -0.8754692 & 0.006 & -0.4834261 & 0.180 & -0.6484108 & 0.000 \\
\hline ninguno & -0.4700031 & 0.000 & -0.7884579 & 0.000 & -0.5877867 & 0.000 \\
\hline bachillerato & 0.1403055 & 0.001 & 0.2159519 & 0.000 & 0.2678795 & 0.000 \\
\hline profesional & 0.7985077 & 0.000 & 1.2039730 & 0.000 & 0.9162912 & 0.000 \\
\hline posgrado & 1.4046430 & 0.000 & 1.8191590 & 0.000 & 1.4759060 & 0.000 \\
\hline _cons & 12.7938600 & 0.000 & 12.4663600 & 0.000 & 12.6313400 & 0.000 \\
\hline \multicolumn{7}{|l|}{ Quantile 50} \\
\hline edad1 & 0.036479 & 0.068 & 0.0379791 & 0.295 & 0.0007763 & 0.928 \\
\hline edad2 & 0.0802813 & 0.000 & 0.0385227 & 0.287 & 0.0054245 & 0.546 \\
\hline edad3 & 0.0802813 & 0.000 & 0.0385227 & 0.287 & 0.0031004 & 0.722 \\
\hline edad4 & 0.0519829 & 0.028 & 0.0385227 & 0.288 & 0.0031004 & 0.727 \\
\hline edad5 & -0.1711607 & 0.070 & -0.4135628 & 0.043 & -0.2309637 & 0.021 \\
\hline edad6 & -0.4588423 & 0.011 & -1.131402 & 0.001 & -0.7615919 & 0.000 \\
\hline $\begin{array}{l}\text { Ningún años de } \\
\text { eduación }\end{array}$ & -0.2623644 & 0.000 & -0.9467812 & 0.000 & -0.4739103 & 0.000 \\
\hline bachillerato & 0.0458097 & 0.002 & 0.0000000 & 1.000 & 0.0023241 & 0.296 \\
\hline profesional & 0.9331131 & 0.000 & 0.8449783 & 0.000 & 0.8447456 & 0.000 \\
\hline posgrado & 1.4554020 & 0.000 & 1.3558030 & 0.000 & 1.4325320 & 0.000 \\
\hline _cons & 13.3327400 & 0.000 & 13.3374700 & 0.000 & 13.3731300 & 0.000 \\
\hline \multicolumn{7}{|l|}{ Quantile 75} \\
\hline edad1 & -0.0618753 & 0.053 & -0.0355511 & 0.253 & -0.0645390 & 0.004 \\
\hline edad2 & 0,1612682 & 0.000 & 0,0289879 & 0.372 & 0,1177826 & 0.000 \\
\hline edad3 & 0,1177826 & 0.000 & 0,0289879 & 0.383 & 0,0622129 & 0.035 \\
\hline
\end{tabular}




\begin{tabular}{|c|c|c|c|c|c|c|}
\hline \multirow{2}{*}{$\begin{array}{l}\text { Variables } \\
\text { edad4 }\end{array}$} & \multicolumn{2}{|c|}{ Hombre } & \multicolumn{2}{|c|}{ Mujer } & \multicolumn{2}{|c|}{ Total } \\
\hline & 0,1177826 & 0.000 & 0,1266327 & 0.028 & 0,1177826 & 0.000 \\
\hline edad5 & $-0,0768089$ & 0.176 & $-0,1045437$ & 0.314 & $-0,1053610$ & 0.050 \\
\hline edad6 & $-0,2876825$ & 0.027 & $-1,0773400$ & 0.003 & $-0,2876825$ & 0.070 \\
\hline $\begin{array}{l}\text { Ningún años de } \\
\text { educación }\end{array}$ & -0.3162336 & 0.000 & -0.7907925 & 0.000 & -0.3215837 & 0.000 \\
\hline bachillerato & 0.0618753 & 0.027 & -0.1335316 & 0.000 & 0.0000000 & 1.000 \\
\hline profesional & 1.091 .495 & 0.000 & 0.9452782 & 0.000 & 0.9808292 & 0.000 \\
\hline posgrado & 1.6713130 & 0.000 & 1.3217550 & 0.000 & 1.4136930 & 0.000 \\
\hline _cons & 13.5923700 & 0.000 & 13.5633800 & 0.000 & 13.5923700 & 0.000 \\
\hline
\end{tabular}

Fuente: diseño del autor según DANE (2015).

En cuanto al cuantil 50 y 75, después de los 72 años de edad, la pérdida del salario de las mujeres supera el $100 \%$. Dicho en otras palabras, dadas las condiciones de vida de sus salarios, en promedio los gastos de las mujeres superan su salario debido a factores como la salud (Granados, 2004).

En cuanto a los años de educación, los resultados son similares a los arrojados en la estimación por MCO; aquí se evidencia que la población -independientemente en qué cuantil se encuentrecon mayor nivel de formación académica siempre tendrá mejoras salariales y mejores cargos en el mercado laboral. Este factor permitirá una propensión marginal al ahorro en la medida que se avance en los niveles educativos, lo que se traduce en un mayor consumo futuro.

\section{Conclusiones}

Con base en el análisis de corte trasversal para el año 2015 se realizó otro exploratorio que demostró que la hipótesis del ciclo vital de Franco Modigliani se cumple para el caso colombiano, con la salvedad de que los salarios de los trabajadores que invierten en educación sigue aumentando conforme avanzan en edad y son más productivos aún en la edad de jubilación.

La educación es una de las variables fundamentales para aumentar las capacidades y la productividad, que se ve reflejada en las mejoras salariales. Además permite aumentar la participación en el mercado de trabajo con respecto a los otros niveles de educación.

Asimismo, los niveles más altos de educación permiten mayor ahorro, dado que la formación posibilita tener más renta disponible. De igual manera, es inevitable el desahorro en las últimas etapas de la vida de las personas. De la misma forma, las mujeres en promedio presentan mayor desahorro en las últimas etapas de la vida con respecto de los hombres. Sin embargo, la preparación en años de educación permite que las mujeres tengan un consumo futuro mayor con respecto al de los hombres.

\section{Referencias}

Departamento Administrativo Nacional de Estadística. (2015). Encuesta nacional de calidad de vida-ECV 2015. Recuperado de http://formularios.dane.gov.co/Anda_4_1/index.php/cata$\log / 419 /$ get_microdata

Dornbusch, R., Fisher, S., y Startz, R. (2009). Macroeconomía. Chicago: McGraw-Hill/Interamericana de España.

Granados, P. (2004). Función de ingresos de los hogares chilenos: ciclo de vida y persistencia de shoks en el tiempo (documento de trabajo n. ${ }^{\circ} 257$ ). Chile: Banco central de Chile. 
JUAN CARLOS MEZA MARTÍNEZ

Hussein, K., y Thirlwall, A. (1999). Explaining diferences in the domestic saving ratio across countries: a panel data study. Kent, Reino Unido: Department of economics, Universidade de Kent.

Mas-Colell, A. (1995). Microeconomic Theory. EE. UU.: Oxford University Press.

McConell, C. (2007). Economía Laboral. España: McGraw-Hill/Interamericana de España.

Melo, L., Zarate, H., y Tellez, J. (2006). El ahorro de los hogares en Colombia. Revista Banco de Republica, 12.

Villar, F. (2012). El enfoque del ciclo vital: hacia un abordaje evolutivo del envejcimiento. En S. Pinazo y M. Sánchez (Eds.), Gerontología: Actualización, innovación y propuestas (pp. 147181). Madrid: Pearson 